\title{
Prevalência da Colonização Vaginal e Anorretal por Estreptococo do Grupo B em Gestantes do Terceiro Trimestre
}

\author{
Prevalence of Vaginal and Anorectal Colonization by Group B Streptococcus in Pregnant Women \\ in the Last Three Months of Gestation
}

Cláudio Beraldo ${ }^{1}$, Angela Sara Jamusse de Brito ${ }^{2}$, Halha Ostrensky Saridakis ${ }^{3}$, Tiemi Matsuo ${ }^{4}$

\begin{abstract}
RESUM0
Objetivos: estudar a prevalência da colonização por estreptococo do grupo B em gestantes do terceiro trimestre, atendidas em um hospital regional, e avaliar a associação da colonização com as variáveis maternas demográficas e clínicas.

Métodos: no período de 8 de outubro de 2002 a 26 de março de 2003, foi realizado um estudo transversal (de prevalência) com 309 gestantes no terceiro trimestre. Amostras de secreção vaginal e anorretal foram coletadas e testadas para identificação presuntiva do estreptococo do grupo B. Foram incluídas as gestantes com gestação maior ou igual a 36 semanas datadas por ultra-sonografia e excluidas as que se recusaram a participar, as em uso de antibioticoterapia e as que haviam sido submetidas a exame ginecológico pelo período mínimo de 24 horas antes da coleta. As gestantes foram caracterizadas por variáveis demográficas (raça, idade, grau de escolaridade, renda familiar e número de gestações) e clínicas (idade gestacional, ocorrência de infecção urinária durante a gestação atual, ruptura prematura de membranas e tempo de bolsa rota, febre materna intraparto, corioamnionite, líquido amniótico com mecônio, via de parto utilizada, febre materna pós-parto e endometrite). Resultados: das gestantes, 46 estavam colonizadas pelo estreptococo do grupo $B$, sendo que 26 (56,5\%) tiveram a cultura vaginal positiva, $8(17,4 \%)$ a cultura anorretal positiva e 12 $(26,5 \%)$ tiveram tanto a cultura vaginal como a retal positivas. Nenhuma das variáveis analisadas neste estudo foi estatisticamente significativa quanto à colonização pelo estreptococo do grupo $B$. Os resultados obtidos foram submetidos à análise bivariada pelo teste do $\chi^{2}$ e teste exato de Fisher quando apropriado.

Conclusão: a taxa de prevalência da colonização vaginal e anorretal pelo estreptococo do grupo B em gestantes no terceiro trimestre, foi de 14,9\%. Não houve associação entre fatores de risco (primigestação, idade materna inferior a 20 anos e nível sócio econômico baixo) e a prevalência da infecção.
\end{abstract}

PALAVRAS-CHAVE: Estreptococo do grupo B. Infecção neonatal. Infecção na gravidez. Rotura prematura de membrana. Prematuridade.

${ }^{1}$ Departamento de Ginecologia e Obstetrícia; ${ }^{2}$ Departamento de Pediatria e Cirurgia Pediátrica; ${ }^{3}$ Departamento de Microbiologia e ${ }^{4}$ Departamento de Matemática Aplicada da Universidade Estadual de Londrina

Correspondência:

Cláudio Beraldo

Avenida Juscelino Kubitscheck 989, apto. 101

86020-000 - Londrina - PR

Tel.: (43) 3323-7498

e-mail: claudioberaldo@uol.com.br

\section{Introdução}

Durante as três últimas décadas, poucos tópicos na literatura científica obstétrica e pediátrica, como também da imprensa leiga, têm despertado tanta atenção como a infecção neonatal de início precoce pelo estreptococo do grupo B 
(Streptococcus agalactiae). Essas infecções apresentam alto grau de morbidade e mortalidade, principalmente nos pacientes com diminuição da defesa imunológica ${ }^{1}$. Os neonatos são afetados com maior freqüência, sendo os prematuros e pequenos para a idade gestacional os que apresentam o maior risco de infecção².

O estreptococo do grupo B é capaz de causar infecções no organismo materno como: cistite, pielonefrite, endocardite, endometrite, celulites, sepse materna puerperal ou não, além de comprometer a evolução da gestação, provocando abortamento, morte fetal intra-uterina, corioamnionite, ruptura prematura de membranas e parto prematuro com conseqüente aumento da incidência de prematuridade. Além disso, pode haver repercussão direta sobre o neonato, como baixo peso ao nascer e infecções no período pós-parto, como: pneumonias, infecções cutâneas, ósseas ou articulares e meningite, podendo causar retardo mental, assim como perda de visão e audição nas crianças sobreviventes ${ }^{2-4}$.

De acordo com a literatura, as taxas de prevalência da colonização por estreptococo do grupo $\mathrm{B}$ podem variar de 5 a $40 \%$, devendo-se essa grande variação aos seguintes fatores: o período de gestação no qual as culturas são realizadas, o local de coleta, os métodos bacteriológicos utilizados para a detecção do estreptococo do grupo B e a origem e características da população estudada ${ }^{5-7}$. Nos estudos brasileiros as taxas têm variado de 4 a $17 \% \%^{5,8,9}$.

Nos Estados Unidos, nos anos 70, o estreptococo do grupo B emergiu como a principal causa infecciosa de morbidade e mortalidade em neonatos, com taxa de mortalidade em torno de 55\%. Em 1990, houve cerca de 7600 casos de sepse neonatal e 310 óbitos $^{10,11}$. Desde 1996, com a instituição das estratégias preventivas, recomendadas pelo CDC (Centers for Disease Control and Prevention), do uso de antibiótico profilático no parto das gestantes de risco para infecção pelo estreptococo do grupo B, houve decréscimo de quase $70 \%$ na incidência da doença estreptocócica de início precoce no recém-nascido, diminuindo de 1,5 para 0,5 por 1000 nascidos vivos ${ }^{4,10,11}$.

No Brasil, o estreptococo do grupo B não tem sido ainda devidamente valorizado na etiologia dos processos infecciosos que acometem os recémnascidos e as puérperas, apesar da gravidade da infecção e de a mesma ser passível de benefícios profiláticos ${ }^{12}$.

O objetivo deste estudo foi determinar a prevalência de gestantes colonizadas pelo estreptococo do grupo B em nosso meio e avaliar a associação da colonização com variáveis maternas demográficas e clínicas, como primeiro passo para a compreensão do problema, além de acrescentar informações à escassa documentação nacional disponível.

\section{Métodos}

De 8 de outubro de 2002 a 26 de março de 2003 foi realizado estudo transversal (de prevalência) da colonização por estreptococo do grupo B (Streptococcus agalactiae) em gestantes com mais de 36 semanas de gestação à ultra-sonografia em trabalho de parto ou não, atendidas na Maternidade Municipal Lucilla Ballalai, em Londrina. Trata-se de maternidade pública, de baixo risco, conveniada com o Sistema Único de Saúde (SUS) e campo de estágio para a Universidade Estadual de Londrina (UEL).

As gestantes foram esclarecidas quanto à finalidade do estudo e, após concordarem em participar e assinarem o termo de consentimento livre e esclarecido, foram submetidas à coleta de duas amostras de secreção para cultura: um swab anorretal e um swab vaginal (no terço distal da vagina). As coletas de material foram realizadas pelo próprio autor $(\mathrm{CB})$. Incluímos todas as gestantes com idade gestacional de 36 ou mais semanas à ultrasonografia que chegavam à maternidade para avaliação obstétrica em trabalho de parto ou não, com bolsa rota ou não. Excluímos as gestantes que se recusaram a participar do estudo (três gestantes), as que estavam em uso de antibiótico e as que haviam sido submetidas a exame ginecológico pelo período de 24 horas antes da coleta de material.

Juntamente com a coleta de material para cultura, foi preenchido formulário com identificação da gestante, com dados de pré-natal e informações adicionais relativas à gestação atual, como: raça, idade, grau de escolaridade, renda familiar, número de gestações, idade gestacional e ocorrência de infecção urinária durante a gestação, como também informações com relação à evolução do trabalho de parto, parto e puerpério: ruptura prematura de membranas e tempo de bolsa rota, febre materna intraparto, corioamnionite, líquido amniótico com mecônio, via de parto utilizada, febre materna pós-parto e endometrite.

Quanto à raça, as gestantes foram estratificadas em brancas e não brancas, e quanto à idade, em menor que 20 anos e maior ou igual a 20 anos. O grau de escolaridade foi dividido em sete categorias: analfabeta, primeiro grau incompleto e completo, segundo grau incompleto e completo, superior incompleto e completo. A renda familiar foi estratificada em até um salário, até dois salários, mais de dois salários e não informada. 
O número de gestações foi dividido em duas ou menos e três ou mais gestações e a idade gestacional em menor que 37 semanas e maior ou igual a 37 semanas. Quanto ao tempo de bolsa rota, o mesmo foi dividido em maior ou igual a 18 horas e menor que 18 horas.

Foi considerado como ocorrência de infecção urinária quando a paciente, durante o prénatal, apresentasse queixas urinárias e exame de rotina do sedimento urinário alterado, com o número de leucócitos acima do normal e a presença ou não de bactérias no material. Não se teve conhecimento do resultado das uroculturas, pois nos cartões de pré-natal das gestantes não havia referência ao resultado das mesmas.

A presença de corioamnionite foi definida como febre maior ou igual a $37,8^{\circ} \mathrm{C}$ durante o trabalho de parto, associada com um ou mais dos seguintes sintomas e sinais: taquicardia materna, taquicardia fetal, líquido amniótico de odor fétido e hemograma materno sugerindo infecção. A endometrite foi caracterizada por: hipertermia, dor e subinvolução uterina e lóquios purulentos e fétidos, podendo ainda estar associada ou não à infecção da ferida operatória.

As amostras de secreção foram coletadas do reto e do terço distal da vagina utilizando-se swabs que foram a seguir inoculados separadamente em meio HPTH: Hitchens - Pike - Todd - Hewitt (Mocelin et al. ${ }^{13}$ ), preparado com infusão de coração de boi fresco, neopeptone (Difco, Detroit), protease peptona, extrato de leveduras (Difco, Detroit), corrigido o $\mathrm{pH}$ para 7,0 com $\mathrm{NaOH}$, e adicionado bicarbonato de sódio, dextrose e $\mathrm{NaCl}$. Após fervura, foi adicionada solução aquosa de cristal de violeta (1:25000) e azida sódica (1:200) e finalmente ágar-ágar (Difco, Detroit). O meio assim preparado foi distribuído em tubos e submetido à esterilização em autoclave por 15 minutos a $120 C^{-}$ e 1 atmosfera de pressão. No momento do uso foi acrescido de $5 \%$ de sangue de coelho desfibrinado (aproximadamente 0,5 mL de sangue de coelho para $10 \mathrm{~mL}$ do meio). As amostras coletadas e inoculadas foram incubadas a $36^{\circ} \mathrm{C}$ por 18 a 24 horas, não tendo sido armazenadas nem refrigeradas antes da incubação. Caso não houvesse crescimento, o mesmo era reincubado por mais 18 a 24 horas.

\section{Identificação do estreptococo do grupo B}

Após a incubação em HPTH, as culturas foram semeadas em ágar sangue de coelho e incubadas a $36^{\circ} \mathrm{C}$ por mais 24 horas, quando então foi realizada a leitura macroscópica das colônias. Estas se caracterizavam por serem diminutas e circundadas por halo de beta hemólise. Em seguida foi realizada a bacterioscopia após coloração de Gram, buscando a presença de cocos gram-positivos dispostos em cadeia e/ou pares e após procedeu-se a identificação presuntiva do grupo.

As colônias que apresentavam morfologia compativel com cocos gram-positivos foram semeadas em ágar BHI (brain - heart - infusion) (Difco, Detroit) e depois testadas quanto à produção de catalase, selecionando-se as negativas (não aparecimento de bolhas), ou seja, o Streptococcus sp.

As cepas identificadas como Streptococcus sp foram ainda testadas quanto ao seu crescimento em meio contendo $\mathrm{NaCl}$ a $6,5 \%$, à prova de CAMP (Christie, Atkins e Munch - Peterson) em ágar sangue de carneiro e hidrólise do hipurato (Difco, Detroit).

As cepas positivas para a prova de CAMP (presença de zona clara em forma de meia lua sobre o ágar sangue de carneiro) e para hidrólise do hipurato (o tubo de ensaio adquire coloração púrpura) foram consideradas Streptococcus agalactiae e estocadas em sangue desfibrinado de coelho para posterior confirmação por meio da grupagem sorológica com antisoro contra grupo B.

\section{Análise estatistica}

Os dados foram coletados seguindo protocolo padronizado e transportados para um banco de dados do Epi-Info, versão 6.04d (janeiro 2001), desenvolvido por The Division of Surveillance and Epidemiology Program Office, Centers for Disease Control and Prevention (CDC), Atlanta, Georgia.

Para a descrição das variáveis utilizaramse tabelas de freqüência simples e percentual. Foi realizada a análise bivariada para avaliar a associação entre gestantes com cultura positiva para o estreptococo do grupo B e as variáveis por meio do teste de $\chi^{2}$ e teste exato de Fisher quando apropriado. Adotou-se o nível de significância de 5\% em todos os testes.

A pesquisa foi previamente submetida à análise e aprovada pelo Comitê de Ética Médica do Hospital Universitário de Londrina em 26 de março de 2001.

\section{Resultados}

Das 309 gestantes que participaram do estudo, 46 apresentaram a cultura vaginal e/ou retal positiva para o estreptococo do grupo $\mathrm{B}$, resultando em uma prevalência de 14,9\% (IC 95\%: 11,2-19,5).

Das 46 gestantes que apresentaram cultura positiva para o estreptococo do grupo B, 26 
$(56,5 \%)$ tiveram a cultura vaginal positiva, 8 $(17,4 \%)$ apresentaram cultura retal positiva e 12 $(26,1 \%)$, ambas as culturas positivas (Tabela 1).

Tabela 1 - Distribuição das gestantes com cultura positiva para o estreptococo do grupo $\mathrm{B}$ de acordo com o local de coleta da amostra.

\begin{tabular}{lcc}
\hline Local & Presença do estreptococo do grupo B \\
& $\mathbf{n}$ & $\%$ \\
\hline Vaginal & 26 & 56,5 \\
Anorretal & 8 & 17,4 \\
Vaginal + Anorretal & 12 & 26,1 \\
Total & 46 & 100,0 \\
\hline
\end{tabular}

A Tabela 2 mostra que, das 46 gestantes com cultura positiva para o estreptococo do grupo B, 38 tiveram a cultura vaginal positiva e apenas 8 , negativa, o que dá uma percentagem de positividade de $82,6 \%$ e de $17,4 \%$ falso-negativo, ou seja, essas 8 gestantes cuja cultura vaginal foi negativa, na verdade, estavam colonizadas, pois a cultura anorretal das mesmas foi positiva para o estreptococo do grupo B. Com relação à cultura anorretal, 20 gestantes a tiveram positiva, ao passo que 26 apresentaram culturas negativas, sendo a percentagem de positividade de $43,5 \%$ e de falsonegativo de $56,5 \%$, e também essas 26 gestantes estavam colonizadas, pois a cultura vaginal foi positiva para o estreptococo do grupo B, mostrando assim, a importância de se realizar a coleta de secreção de ambos os locais visando uma maior taxa de prevalência.

Tabela 2 - Positividade e resultados falsos-negativos de acordo com o local para as 46 amostras positivas para o estreptococo do grupo B.

\begin{tabular}{lcccc}
\hline Local & $\begin{array}{c}\text { Estreptococo do Grupo B } \\
\text { Positivos }\end{array}$ & $\begin{array}{c}\text { Positivos (\%) } \\
\text { Negativos }\end{array}$ & $\begin{array}{c}\text { Falso- } \\
\text { negativos (\%) }\end{array}$ \\
\hline Vaginal & 38 & 8 & 82,6 & 17,4 \\
Anorretal & 20 & 26 & 43,5 & 56,5 \\
\hline
\end{tabular}

Ao analisarmos a variável idade, observamos que a faixa mais prevalente foi a de 20 anos ou mais, com 239 gestantes (77,3\%). Dessas, $36(15,1 \%)$ apresentaram cultura positiva para o estreptococo do grupo $B$, que não diferiu significantemente $(p=0,8724)$, da freqüência de 10 gestantes $(14,3 \%)$ encontrada na faixa etária com menos de 20 anos.

Guanto à raça, houve predomínio da branca, com 243 gestantes (78,6\%), e dessas, 37 (15,2\%) tinham cultura positiva, e entre as da raça não branca, $9(13,6 \%)$ apresentaram este resultado, não ocorrendo diferença significante $(\mathrm{p}=0,7476)$. Quanto ao número de gestações prévias, 202 gestantes $(65,4 \%)$ tinham duas ou menos gestações e dessas, $28(13,9 \%)$ apresentaram cultura positiva. Dentre as gestantes com três ou mais gestações, $18(16,8 \%)$ tinham cultura positiva, sendo que essas proporções não foram estatisticamente diferentes $(\mathrm{p}=0,4866)$.

Quanto à idade gestacional, das 16 gestantes $(5,2 \%)$ com idade gestacional menor que 37 semanas, $3(18,8 \%)$ tiveram a cultura positiva, o mesmo ocorrendo entre as 43 gestantes (14,7\%) com idade gestacional igual ou maior a 37 semanas. Esta diferença também não foi significante $(p=0,7157)$.

Das 73 gestantes $(23,6 \%)$ que apresentaram pelo menos um episódio de infecção urinária durante a gestação, $15(20,5 \%)$ tinham a cultura positiva. Essa proporção não diferiu significativamente $(p=0,1200)$ da encontrada entre as gestantes sem infecção urinária e com cultura positiva $(13,1 \%)$.

A ruptura prematura de membranas (ruptura do âmnio antes do início do trabalho de parto, independentemente da idade gestacional) ocorreu em 27 gestantes (8,7\%). Dessas, 7 (25,9\%) tinham cultura positiva, não diferindo estatisticamente das gestantes que não tiveram ruptura prematura de membranas e tinham cultura positiva $(p=0,0962)$.

Das gestantes com cultura positiva, 2 $(22,2 \%)$ tiveram tempo de bolsa rota maior ou igual a 18 horas, e $44(14,7 \%)$, menos de 18 horas. Essas freqüências não apresentaram diferença estatisticamente significante $(\mathrm{p}=0,6273)$.

Também com relação às demais variáveis não encontramos diferença estatisticamente significativa no estudo de gestantes colonizadas ou não (Tabela 3).

Tabela 3 - Frequêencia dos fatores de risco para presença do estreptococo do grupo B nas 309 gestantes estudadas.

\begin{tabular}{|c|c|c|c|c|c|}
\hline \multirow{4}{*}{ Fatores de risco } & \multirow{2}{*}{\multicolumn{4}{|c|}{$\begin{array}{c}\text { Presença do estreptococo } \\
\text { do grupo B }\end{array}$}} & \multirow{4}{*}{ Valor de $\mathrm{p}$} \\
\hline & & & & & \\
\hline & \multicolumn{2}{|c|}{ Sim } & \multicolumn{2}{|c|}{ Não } & \\
\hline & $\mathrm{n}$ & $\%$ & $\mathrm{n}$ & $\%$ & \\
\hline Idade (<20 anos) & 10 & 21,7 & 60 & 22,8 & $0,8724^{a}$ \\
\hline Raça (não branca) & 9 & 19,6 & 57 & 21,7 & $0,7476^{a}$ \\
\hline \multicolumn{6}{|l|}{ Grau de escolaridade } \\
\hline (analfabeta e $1^{\circ}$ grau incompleto) & 29 & 63,0 & 131 & 49,8 & $0,0975^{a}$ \\
\hline Renda familiar (até 2 salários) & 25 & 54,3 & 114 & 43,3 & $0,1664^{a}$ \\
\hline Paridade ( $\leq 2$ gestações) & 28 & 60,9 & 174 & 66,2 & $0,4866^{a}$ \\
\hline Tipo de parto (vaginal) & 29 & 63,0 & 182 & 69,2 & $0,7476^{a}$ \\
\hline Idade gestacional (IG <37 sem) & 3 & 6,5 & 13 & 4,9 & $0,7156^{b}$ \\
\hline Tempo de bolsa rota ( $\geq 18$ horas) & 2 & 4,3 & 7 & 2,7 & $0,6272^{b}$ \\
\hline Febre materna intraparto & 1 & 2,2 & 3 & 1,1 & $0,4770^{b}$ \\
\hline Corioamnionite & 1 & 2,2 & 3 & 1,1 & $0,4770^{b}$ \\
\hline Total & 46 & 14,9 & 263 & 85,1 & \\
\hline
\end{tabular}

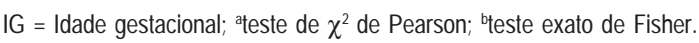




\section{Discussão}

Por ser tão evidente a relação entre a presença do Streptococcus agalactiae na flora vaginal da gestante e a possibilidade de colonização do recém-nascido, torna-se necessário como primeiro passo para o estudo e compreensão do problema, determinar a percentagem de gestantes colonizadas em nosso meio, conforme foi objetivo deste estudo.

Em nosso estudo encontramos que a taxa de colonização por estreptococo do grupo B nas gestantes está em concordância com as taxas encontradas em outros estudos. Uma de cada quatro ou cinco mulheres grávidas traz o estreptococo do grupo B no reto ou vagina, sendo a taxa de prevalência da colonização por estreptococo do grupo B variável de 5 a $40 \%^{14-16}$.

Os diferentes estudos mostram como as taxas de colonização podem variar segundo a origem, as características da população estudada e o modo de obtenção do material para cultura. Em estudo prospectivo realizado em quatro hospitais de Houston com 546 mulheres, mostrou-se que 153 (28\%) estavam colonizadas; num estudo realizado no Flórida Hospital Family Practice Residency Program com 222 pacientes de 35 a 37 semanas, 54 pacientes $(24,3 \%)$ tiveram culturas positivas para o estreptococo do grupo $\mathrm{B}^{16,17}$.

Kieran et al. ${ }^{8}$ e Grimwood et al. ${ }^{14}$ relataram, em estudo realizado nas Community and Hospital Antenatal Clinics em Wellington e Auckland, que de 240 mulheres entre 35 a 37 semanas de gestação, 52 mulheres (22\%) estavam colonizadas pelo estreptococo do grupo B. A taxa de prevalência de colonização em mulheres irlandesas foi de 25,6\%, segundo um estudo do Hospital e Maternidade Municipal de Dublin.

Em nosso país, segundo Rocha et al. ${ }^{15}$, Oliveira et al. realizando trabalho com 100 gestantes no terceiro trimestre de gravidez, atendidas no Serviço de Obstetrícia do Hospital Santa Lúcia de Brasília, encontraram a taxa de colonização de $4 \%$. No Serviço de Pré-Natal do Hospital Gastroclínica (FIMADEN) - São Paulo, a taxa de colonização foi de $17 \%^{8}$.

Em nosso meio, Mocelin et al. ${ }^{13}$, em estudo de 100 gestantes atendidas no ambulatório e/ou Pronto Socorro Obstétrico do Hospital Universitário Regional do Norte do Paraná, Londrina, encontraram a taxa de colonização de 15\%.

A taxa de colonização por estreptococo do grupo B aumenta de 5 a $27 \%$, quando a amostra é coletada de mais de um local. A obtenção de cultura da cérvix ao invés da vagina inferior e reto pode falhar na identificação de mais da metade das mulheres colonizadas pelo estreptococo do grupo $B$, assim optamos pela coleta de amostras de ambos os locais, trato genital inferior e anorretal, possibilitando um resultado mais fiel.

De acordo com Baker e Edwards ${ }^{16}$, Badri et al. foram os primeiros a investigar a hipótese de Aber et al. de que o local primário de infecção era o trato gastrointestinal inferior e que a colonização genital meramente refletia contaminação anorretal. Eles detectaram taxas de isolamento mais elevadas de culturas retais (17\%) do que de culturas vaginais $(10,2 \%)^{6}$.

O terço distal da mucosa vaginal pode estar colonizado pelo estreptococo do grupo B do canal anorretal. Por esta razão, swabs diagnósticos são direcionados para essas áreas ${ }^{18}$. O material da vagina distal e reto coletado entre a $35^{\mathrm{a}}$ e a $37^{\mathrm{a}}$ semana de gestação, semeado em meio caldo seletivo e submetido a procedimentos específicos para cultura e caracterização do estreptococo do grupo $\mathrm{B}$ identificará a maioria das mulheres colonizadas, sendo portanto esta a melhor forma de predição do estado de colonização durante o trabalho de parto. Para diminuir a incidência da infecção neonatal pelo estreptococo do grupo B, os protocolos de prevenção têm incluído a obtenção de culturas vaginal e anorretal, tratando-se as pacientes cujas culturas vaginal e/ou anorretal são positivas.

Em nosso estudo constatamos que oito gestantes $(17,4 \%)$ que tiveram somente a cultura retal positiva não receberiam quimioprofilaxia de prevenção de sepse neonatal precoce, caso o mate-rial retal para cultura não tivesse sido coletado.

É importante estar atento às diferenças cruciais na composição das diferentes populações, que podem apresentar muitas das situações citadas como fatores de risco para doença pelo estreptococo do grupo $\mathrm{B}^{14}$. Nos vários estudos de prevalência do estreptococo do grupo B a taxa de colonização é maior nas primigestas que em multíparas (três ou mais gestações), em mulheres com status socioeconômico baixo, em mulheres com idade inferior a vinte anos (a correlação entre a concentração de anticorpos maternos e idade materna pode explicar a associação epidemiológica), nas mulheres negras em relação às mulheres de outros grupos étnicos, e nas mulheres diabéti$\operatorname{cas}^{6,7,19,20}$. Nosso estudo não demonstrou associação significativa entre estes fatores e a presença da bactéria.

O status de defesa do feto também é fator adicional que aumenta o risco de transmissão do estreptococo do grupo B, pois uma criança pré- 
têrmo tem menos capacidade para resistir aos organismos invasivos, tendo maior chance para desenvolver a doença. A ruptura prolongada de membranas (maior ou igual a 18 horas), corioamnionite, febre materna intraparto (temperatura maior ou igual a $38^{\circ} \mathrm{C}$ ), trabalho de parto prematuro (menor que 37 semanas de gestação), infecção urinária ou bacteriúria pelo estreptococo do grupo B na gestação atual também são fatores de risco para a aquisição do estreptococo do grupo $\mathrm{B}^{21}$.

Esses achados não foram significativos neste estudo, pois somente 16 recém-nascidos nasceram prematuramente e 9 tiveram bolsa rota por período maior ou igual a 18 horas. $O$ índice de prematuridade neste estudo foi de $5,2 \%$, diferente do município de Londrina, que é de 8,3\% (SINASC - 2002), e as gestantes foram de uma maternidade de baixo risco, com taxa de prematuridade menor.

Das gestantes incluídas no estudo, 73 (23,6\%) relataram ter tido infecção urinária durante a gestação. Embora não tivéssemos tido acesso ao resultado das uroculturas, valorizamos essa informação do cartão de pré-natal, visto que o aumento aumento da infecção urinária pelo estreptococo do grupo B tem sido associado ao aumento da prevalência de parto prematuro e ruptura prematura de membranas ${ }^{22}$.

A epidemiologia da doença estreptocócica em mulheres grávidas e neonatos tem sido extensivamente estudada nos Estados Unidos e Europa, mas o mesmo não se observa na América Latina. Devido ao elevado risco de morte, é necessário prevenir a doença invasiva pelo estreptococo do grupo B e muitos estudos têm mostrado que são necessários diagnóstico e tratamento materno adequados para reduzir a transmissão vertical do estreptococo do grupo B e a sepse neonatal de início precoce. Nos Estados Unidos da América, a redução na sepse neonatal na última década tem sido atribuída a quimioprofilaxia durante o trabalho de parto para mulheres que apresentam o estreptococo do grupo B na urina, vagina e/ou reto $^{18}$.

A determinação dos fatores de risco por meio da anamnese da paciente e exames clínicos acompanhados de culturas de urina, vaginal e retal para o estreptococo do grupo B deve ser estimulada entre os profissionais de saúde responsáveis pelos cuidados de pré-natal. Isso permitiria a identificação de mulheres colonizadas pelo estreptococo do grupo B e a instituição de profilaxia antibiótica endovenosa durante o trabalho de parto, a qual certamente reduziria a infecção neonatal.
Ressaltamos, assim, a importância de cada serviço de pré-natal implantar um esquema de coleta de secreção vaginal e anorretal das gestantes entre a 35 a e a $37^{a}$ semana de gestação para identificação do estreptococo do grupo B, bem como utilizar rotina de profilaxia antibiótica endovenosa com penicilina cristalina ou ampicilina para as gestantes em trabalho de parto que tenham cultura vaginal e/ou anorretal positivas para o estreptococo do grupo B. Pacientes com culturas não realizadas ou não prontas receberiam profilaxia intraparto se o parto ocorrer antes da 37ª semana de gestação, se o período após a ruptura das membranas for maior ou igual a 18 horas ou se a temperatura intraparto for maior ou igual a $38^{\circ} \mathrm{C}$.

\section{ABSTRACT}

Purpose: to study the prevalence of colonization by group $B$ Streptococcus in pregnant women in the last three months of gestation, and to evaluate the association of colonization with demographic and clinical maternal variables.

Methods: from October 8, 2002 to March 26, 2003, a transversal study of prevalence of colonization by group $B$ Streptococcus was carried out in 309 pregnant women in the last three months of gestation. Samples of vaginal and anorectal secretions were collected and were tested for presumptive identification of group B Streptococcus. The pregnant women were studied according to race, age, level of instruction, family income, number of gestations, gestational age, history of urinary tract infection during present pregnancy, premature rupture of membranes and duration of ruptured membranes, intrapartum fever, chorioamnionitis, meconium in the amniotic fluid, spontaneous or cesarean section delivery, postpartum fever, and postpartum endometritis.

Results: fourty-six pregnant women were diagnosed with group B Streptococcus: 26 of them (56.5\%) had positive vaginal culture, $8(17.4 \%)$ positive anorectal culture and $12(26.5 \%)$ had both vaginal and anorectal positive cultures. None of the maternal variables were statistically significant with respect to group B Streptococcus colonization. The results were submitted to $\chi^{2}$ bivariate analysis and, when appropriate, Fisher's exact test.

Conclusion: the prevalence rate of vaginal and anorectal colonization by group B Streptococcus in pregnant women in the last three months of gestation, in Londrina - Paraná, was $14.9 \%$.

KEYWORDS: Group B Streptococcus. Neonatal infection. Infection in pregnancy. 


\section{Referências}

1. Caiaffa Filho HH, Mendes CMF. Novo método para a rápida identificação presuntiva dos estreptococos beta-hemolíticos do Grupo B (Streptococcus agalactiae). Rev Bras Patol Clín 1986; 22:43-6.

2. Freitas F, Martins-Costa SH, Ramos JGL, Magalhães JA. Rotinas em obstetrícia. $4^{a}$ ed. Porto Alegre: Artmed; 2002.

3. Grassi MS, Diniz EMA, Vaz FAC. Métodos laboratoriais para diagnóstico da infecção neonatal precoce pelo Streptococcus beta hemolítico do grupo B. Pediatria (São Paulo) 2001; 23:232-40.

4. Cole D, Bernstein PS. An update on perinatal group B streptococcal disease. OB/Gyn Women's Health [serial online] 2002; [cited 2002 Nov 28]; 7(2). Available from: URL: http://www.medscape.com

5. CDC. Centers for Disease Control and Prevention. Decreasing incidence of perinatal group B Streptococcal disease - United States, 1993 -1995. Can Commun Dis Rep [serial online] 1997; [cited 1999 Jun 4]; 23:109-12. Available from: URL: http:// www.medscape.com/govmt/CDC

6. Schuchat A, Whitney C, Zangwill K. Prevention of perinatal group B streptococcal disease: a public health perspective. Proceedings of the American Psychiatric Association Annual Meeting; 1999 May 15-20; Washington, DC, USA. Washington, DC: American Psychiatric Association; 1999.

7. Rocha JES, Tomaz ACP, Rocha DB, et al. Morbidade materna e morbimortalidade perinatal associada à infecção ascendente na rotura prematura das membranas. Rev Bras Ginecol Obstet 2002; 24:15-20.

8. Kieran E, Matheson M, Mann AG, Efstratiou AA, Butler K, Gorman W. Group B Streptococcus (GBS) colonisation among expectant Irish mothers. Ir Med J 1998; 91:21-2.

9. Beardsall K. Guidelines for Group B streptococcus. Arch Dis Child Fetal Neonatal Ed 2001; 84:F77-8.

10.Nascimento DJ. Profilaxia da transmissão vertical do GBS (Streptococco Beta Hemolítico): quando e como tratar. Rev SOGIPA 2002; 22:5.

11.Brumund TT, White CB. An update on group B streptococcal infections in the newborn: prevention, evaluation, and treatment. Pediatr Ann 1998; 27:495-501.
12. Quinlan JD, Hill DA, Maxwell BD, Boone S, Hoover F, Lense JJ. The necessity of both anorectal and vaginal cultures for group B streptococcus screening during pregnancy. J Fam Pract 2000; 49:447-8.

13. Mocelin CO, Carvalho DAF, Brites C, et al. Isolamento de Streptococcus agalactiae de gestantes na Região de Londrina-PR. Rev Bras Ginecol Obstet 1995; 17:915-8.

14.Grimwood K, Stone PR, Gosling IA, et al. Late antenatal carriage of group B Streptococcus by New Zealand women. Aust N Z J Obstet Gynaecol 2002; 42:182-6.

15. Rocha MLF, Beltrame LMR, Guedes SC, et al. Prevalência do Streptococcus agalactiae na flora vaginal de gestantes do último trimestre. Rev Bras Patol Clín 1984; 20:110-2.

16.Baker CJ, Edwards MS. Group B streptococcal infections. In: Remington JS, editor. Infections Diseases of the Fetus of Newborn Infant. $4^{\text {th }}$ ed. Philadelphia: W.B. Saunders; 1995. p. 980-1054.

17.Vaciloto E, Richtmann R, Costa HPF, Kusano EJU, Almeida, MFB, Amaro ER. A survey of the incidence of neonatal sepsis by group B Streptococcus during a decade in a Brazilian maternity hospital. Braz J Infect Dis 2002; 6:55-62.

18. Rowen JL, Baker CJ. Group B streptococcal infections. In: Feigin RD, editor. Textbook of Pediatric Infections Diseases. $4^{\text {th }}$ ed. Philadelphia: W.B. Saunders; 1998. v.1, p. 1089-106.

19.Schuchat A. Epidemiology of group B streptococcal disease in the United States: shifting paradigms. Clin Microbiol Rev 1998; 11:497-513.

20.Edwards MS, Baker CJ. Streptococcus agalactiae (Group B Streptococcus). In: Mandell D, editor. Bennett's Principles and Practice of Infections Diseases. $5^{\text {th }}$ ed. Philadelphia: Churchill-Livingston; 2000. v.2, p. 2156-67.

21.Seo K, McGregor JA, French JI. Preterm birth is associated with increased risk of maternal and neonatal infection. Obstet Gynecol 1992; 79:75-80.

22.McKenna DS, Iams JD. Group B streptococcal infections. Semin Perinatol 1998; 22:267-76. 